\title{
Early post-LASIK flap amputation in the treatment of aggressive, branching keratitis: a case report
}

\author{
Amputação precoce de pedículo de LASIK para o tratamento de ceratite ramificada agressiva: relato de caso
}

John Au ${ }^{1}$, Thomas Plesec ${ }^{1}$, Karolinne Rocha $^{2}$, William Dupps JR. ${ }^{1}$, Ronald Krueger ${ }^{1}$

\begin{abstract}
Infectious keratitis is rare following laser vision correction. We present a case of aggressive fungal keratitis caused by Aspergillus flavus, following laser in situ keratomileusis (LASIK) in the setting of a unique environmental risk factor. We describe the key features of the acute case presentation, which guided empirical medical and surgical treatment, resulting in the most favorable outcome found in the literature, to date.
\end{abstract}

Keywords: Keratomileusis, laser in situ/adverse effects; Keratitis/etiology

\section{RESUMO}

Ceratites infecciosas são raras após a correção da visão a laser. Apresentamos um relato de caso de uma ceratite fúngica agressivo por Aspergillus flavus, após ceratomileuse a laser "in situ" (LASIK) em situação peculiar de fator de risco ambiental. Nós descrevemos as principais características da apresentação caso agudo, que orientou tratamento médico e cirúrgico empírico, demonstrando resultado mais favorável do que o encontrado na literatura até o momento.

Descritores: Ceratomileuse assistida porexcimer laser in situ/efeitos adversos; Ceratite/ etiologia

\section{INTRODUCTION}

Infectious keratitis following laser vision correction is uncommon ${ }^{(1,2)}$ The estimated incidence as reported by the 2008 American Society of Cataract and Refractive Surgery (ASCRS) survey was 19 of 20,941 eyes $(0.09 \%)$. The most commonly cultured organism in this series was methicillin-resistant Staphylococcus aureus. One case was confirmed to be caused by a fungal infection ${ }^{(1)}$. We wish to share our experience with a case of Aspergillus flavus keratitis following laser in situ keratomileusis (LASIK), in association with a potential environmental risk factor.

\section{CASE REPORT}

A 24-year-old female with low myopia was treated with wavefront-optimized femtosecond-LASIK on the WaveLight FS-200 and Allegretto Eye-Q laser platform (Alcon, Fort Worth, TX, USA). Her initial manifest refraction was $-1.25 \mathrm{D}$ sphere in both eyes. Central corneal thickness was $508 \mu \mathrm{m}$ in OD and $505 \mu \mathrm{m}$ in OS, as measured with ultrasound pachymetry. The remainder of her ophthalmic examination and corneal tomography were normal. Her history included prior right V1 dermatome herpes zoster without ophthalmic involvement. The LASIK procedure was uneventful, and she was started on ciprofloxacin 0.3\% (Ciloxan ${ }^{\circledR}$ ) and dexamethasone 0.1\% drops four times daily. On postoperative day 1, the uncorrected distance visual acuity (UDVA) was 20/15 in each eye, and the ophthalmic exam was unremarkable.

On postoperative day 2, the patient experienced severe pain, photophobia, and decreased vision in the right eye. UDVA was 20/50 in the right eye and 20/15 in the left eye. Ophthalmic exam with a slit lamp revealed a dense, branching, feathery infiltrate within the flap interface of the right eye (Figure 1). Given this appearance, the clinical suspicion for fungal keratitis was high.

The patient provided informed consent, and was immediately taken to the operating room for flap-lift, culture, irrigation of the flap interface, and potential flap amputation. The flap was lifted and a gelatinous branching infiltrate was observed, extending superficially into the flap and posteriorly into the stromal bed. After bacterial and fungal culture samples were obtained, the interface was extensively irrigated with a balanced salt solution. The infiltrate penetrated into the flap stroma, and because of the rapid progression $(<24 \mathrm{~h})$ of fungal keratitis, a decision was made to amputate the flap. Hourly topical fortified tobramycin $(13.5 \mathrm{mg} / \mathrm{ml})$, vancomycin $(25 \mathrm{mg} / \mathrm{ml})$, and voriconazole (1\%) were immediately started. Although the infiltrate was clearly in the flap interface and not involving the overlying epithelium, $1 \mathrm{~g}$ of oral valacyclovir three times daily was also started for 10 days because of the patient's history of right $\mathrm{V} 1$ herpes zoster. Prednisolone therapy was discontinued.

The infiltrate remained prominent on the day following flap amputation. The patient was followed-up very closely for the first 3 weeks postflap amputation. Four days postflap amputation, UDVA was 20/100-1. The infiltrate was improving and the epithelium had healed approximately $85 \%$ over the stromal bed (Figure 2 A). Bacterial cultures from the samples taken during flap amputation were negative. Nine days after these were taken, fungal cultures showed A. flavus growth. Histopathology of the flap showed fungal elements within the undersurface of the flap stroma (Figure 3). Fungal sensitivities were requested and hourly topical voriconazole was continued, while the fortified vancomycin and tobramycin were tapered. On day 11 postflap amputation, UDVA in the right eye had improved to 20/40. Photography (Figure 2 B) and corneal SD-OCT (Figure 4) were
Submitted for publication: December 11, 2014

Accepted for publication: February 2, 2015

${ }^{1}$ Cleveland Clinic Foundation, Cole Eye Institute, Cleveland, $\mathrm{OH}$

${ }^{2}$ Medical University of South Carolina, Storm Eye Institute.
Funding: No specific financial support was available for this study.

Disclosure of potential conflicts of interest: None of the authors have any potential conflicts of interest to disclose.

Corresponding author: John Au. 3.501 Jamboree Road \#1.100 - Newport Beach, CA 92660 E-mail: johnau79@gmail.com 
repeated at that time. Antifungal sensitivity testing confirmed that voriconazole was appropriate for the isolated strain (Table 1).

After 3 weeks of hourly voriconazole, UDVA had improved to $20 / 25(-2)$ in the right eye. The manifest refraction was -0.50 D sphere

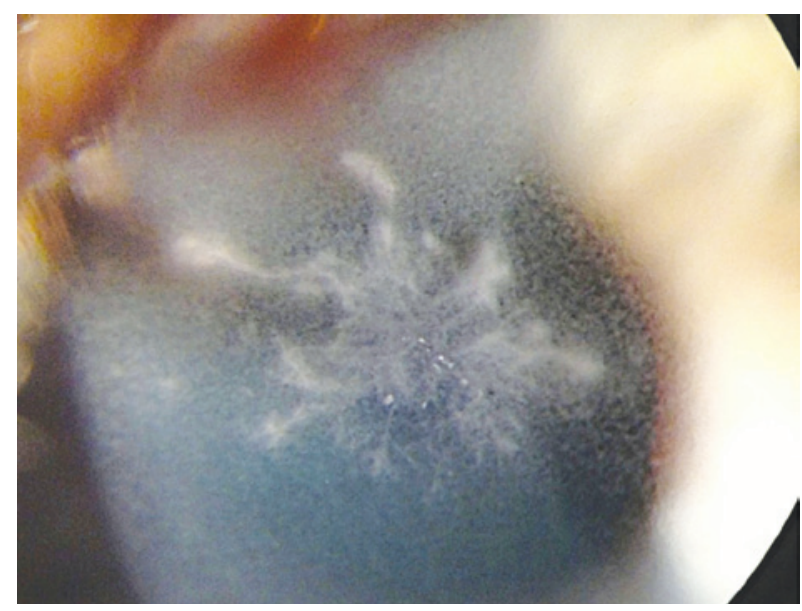

Figure 1. The day 2 postoperative appearance of branching feathery interface infiltrate
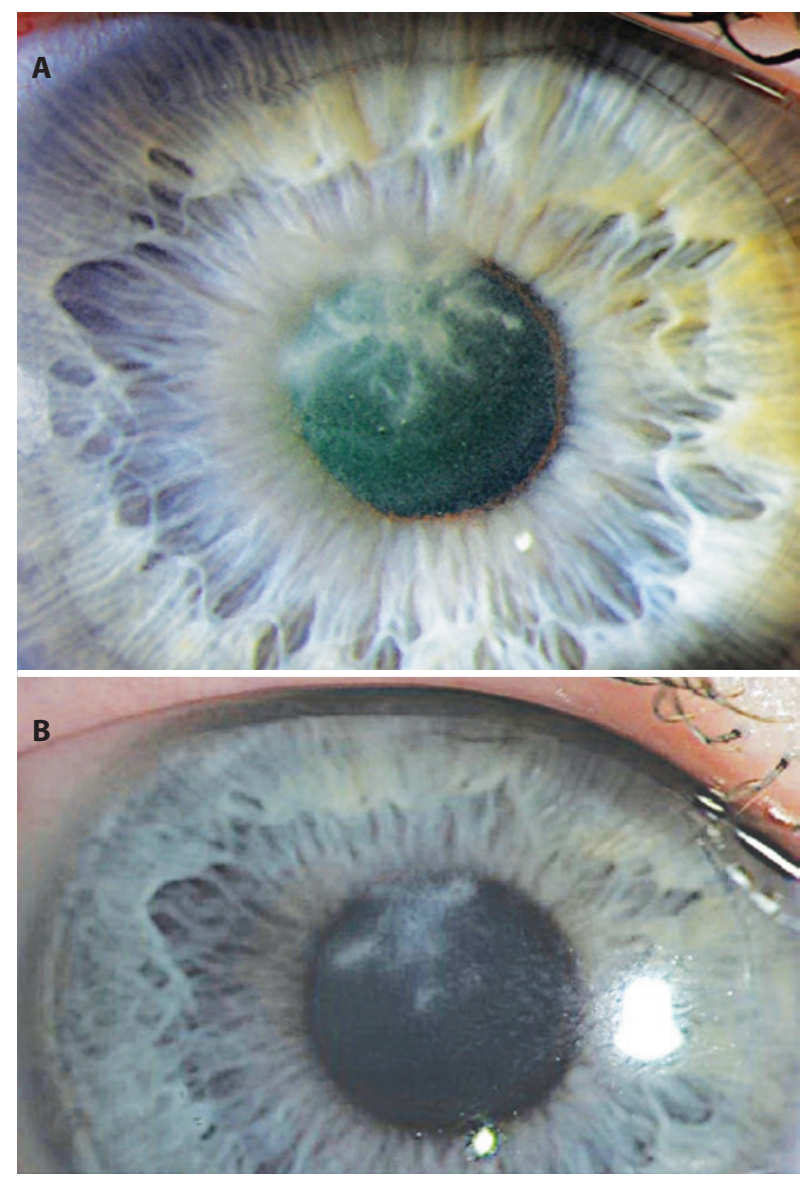

Figure 2. A) The day 2 postflap amputation slit lamp appearance with reformation of dense feathery infiltrate. B) The day 11 postflap amputation slit lamp appearance showing improvement of the infiltrate with decreasing density. with a corrected distance visual acuity of 20/20-3. Voriconazole was tapered and prednisolone acetate was slowly added in an attempt to decrease corneal haze. At 5 months postoperatively, UDVA was $20 / 15$ in the right eye. Ophthalmic exam at that time showed a persistent but improved $1+$ corneal haze. She has not required further intervention to date.

\section{DISCUSSION}

A review of the literature shows a generally poor outcome for post-LASIK Aspergillus keratitis ${ }^{(2-4)}$. Of the published cases, one required a penetrating keratoplasty ${ }^{(2)}$; another resulted in corneal perforation, requiring glue patching ${ }^{(3)}$; and the third resulted in a final pinhole vision of $20 / 40^{(4)}$. Our case demonstrated an aggressive nature of $A$. flavus keratitis, which resulted in lifting of the flap and irrigation of the

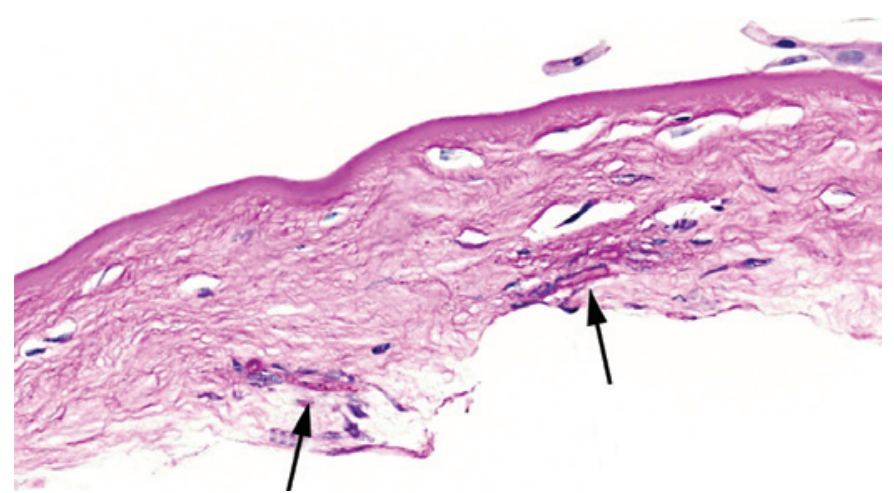

Figure 3. Histology of the amputated corneal flap showing fungal elements in the stroma (black arrows).

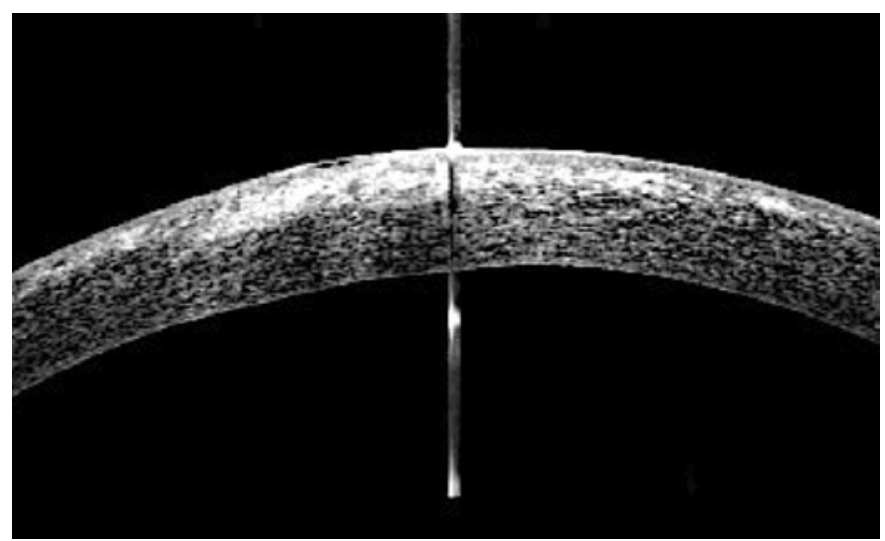

Figure 4. A day 11 postflap amputation SD-OCT image showing dense central hyper-reflectivity, representing the residual infiltrate and surrounding haze. Reepithelialization is noted.

Table 1. The minimum inhibitory concentration (MIC) of antifungal agents for use against Aspergillus flavus cultured in this patient

\begin{tabular}{lc}
\hline Antifungal agent & MIC $(\boldsymbol{\mu g} / \mathbf{m l})$ \\
\hline Amphotericin B & 2 \\
Natamycin & 32 \\
Fluconazole & $>64$ \\
Itraconazole & 1 \\
Posaconazole & 0.5 \\
Voriconazole & 1 \\
\hline
\end{tabular}


interface, as well as complete amputation of the flap. Ultimately, this decision in conjunction with proper empirical medical intervention has allowed for a remarkably good visual result for our patient.

During the week prior to this case, a new wall was built within the refractive suite waiting area, which was not directly connected with the laser suite. However, on the day prior to the case, construction workers had removed some ceiling tiles in the laser suite, in preparation for the upcoming construction and removal of an internal wall.

It is well established in the literature that construction is a risk factor for aerosolized dissemination of spore-forming organisms including fungi ${ }^{(5-9)}$. Several cases and outbreaks of Aspergillus in association with hospital construction have been described, resulting in pulmonary aspergillosis in immunocompromised patients. Primarily, $A$. flavus and A. fumigatus are the responsible organisms in these cases $^{(6-9)}$. A prior outbreak of Aspergillus endophthalmitis has been described in association with hospital construction ${ }^{(5)}$. To the best of our knowledge, this is the first reported case of post-LASIK A. flavus keratitis with construction in the laser suite as a potential risk factor.

Although the presentation of this patient was early for fungal infection, the steroids administered to the patient could have allowed for a more rapid progression of the fungal infection. In addition, we considered that the recent construction in the laser suite could have released fungal organisms. We also considered bacterial, including mycobacterial, and herpetic etiologies. Given the rapid progression, the possibility of bacterial infection remained reasonably high. Therefore, fortified antibiotics were prescribed on an hourly basis from the day of flap amputation until the cultures proved fungal etiology. Atypical mycobacterium should always be considered in LASIK-related infections. However, these infections typically present weeks after the procedure. Finally, we empirically treated the patient for herpetic disease as well because she had a history of herpes zoster. The location of the infiltrate was within the flap interface and not intraepithelial; this led us to favor a fungal or bacterial etiology over a herpetic one.

One valuable clinical lesson gained from this experience is to avoid surgical cases in the setting of recent construction. We are unaware of a specific recommended time period to wait to perform further refractive surgery following such construction events. It is encouraged to cover or move equipment prior to construction. In addition, after construction is complete, a thorough cleaning of all dust-covered surfaces in the area is necessary prior to any further surgery.
A second equally important lesson focuses on trusting clinical judgment based upon physical findings in the absence of more definitive data (i.e., cultures). The initial clinical appearance must guide therapy when more definitive data is lacking. Based on the earliest finding on slit lamp exam, we surmised that flap amputation was indicated, which ultimately led to a good rather than poor postoperative outcome. Several considerations led to this decision: 1) Penetration of the antibiotics and antifungal medication to the infiltrate would be improved. 2) The patient had a low myopic correction with robust residual stromal bed thickness for possible future refractive treatment. 3) The flap was created with a femtosecond laser; therefore, it had a uniform planar shape when amputated, allowing for minimal refractive change when compared with a meniscus-shaped microkeratome flap. These factors, along with prompt, appropriate medical therapy, contributed to this most favorable reported outcome in post-LASIK fungal keratitis.

\section{ACKNOWLEDGMENTS}

Thank you to University of Texas Health Science Center at San Antonio, Department of Pathology for antifungal susceptibility testing.

\section{REFERENCES}

1. Solomon R, Donnenfeld ED, Holland EJ, Yoo SH, Daya S, Güell JL, Mah FS, Scoper SV Kim T. Microbial keratitis trends following refractive surgery: Results of the ASCRS Infectious keratitis survey and comparisons with prior ASCRS surveys of infectious keratitis following keratorefractive procedures. J Cataract Refract Surg. 2011;37(7): 1343-50.

2. Sridhar M, Garg P, Bansal A, Gopinathan U, Aspergillus flavus Keratitis After Laser in Situ Keratomileusis. Am J Ophthalmol. 2000;129(6):802-4

3. Kuo I, Margolis T, Cevallos V, Hwang D, Aspergillus fumigatus Keratitis After Laser In Situ Keratomileusis. Cornea. 2001:20(3):342-4.

4. Sun Y, Jain A, Ta C, Aspergillus fumigatus keratitis following laser in situ keratomileusis J Cataract and Refract Surg. 2007;33(10):1806-7.

5. Tabbara KF, al Jabarti AL. Hospital construction-associated outbreak of ocular aspergillosis after cataract surgery. Ophthalmology. 1998;105(3):522-6.

6. Krasinski K, Holzman RS, Hanna B, Greco MA, Graff M, Bhogal M. Nosocomial fungal infection during hospital renovation. Infect Control. 1985;6(7):278-82.

7. Arnow PM, Andersen RL, Mainous PD, Smith EJ. Pulmonary aspergillosis during hospital renovation. Am Rev Respir Dis. 1978;118(1):49-53.

8. Vonberg RP, Gastmeier P. Nosocomial aspergillosis in outbreak settings. J Hosp Infect. 2006;63(3):246-54

9. Lentino JR, Rosenkranz MA, Michaels JA, Kurup VP, Rose HD, Rytel MW. Nosocomial aspergillosis: a retrospective review of airborne disease secondary to road construction and contaminated air conditioners. Am J Epidemiol. 1982;116(3):430-7. 